\title{
Increased Mesenchymal Stem Cell Functionalization in Three-Dimensional Manufacturing Settings for Enhanced Therapeutic Applications
}

OPEN ACCESS

Edited by:

Elizabeth R. Balmayor, MERLN Institute for Technology-Inspired Regenerative Medicine, Maastricht University, Netherlands

Reviewed by:

Vanessa L. S. LaPointe, Maastricht University, Netherlands Patrina Su Ping Poh, Charité - Universitätsmedizin Berlin,

Germany

*Correspondence:

Dimitrios Kouroupis dxk504@med.miami.edu

Specialty section:

This article was submitted to Preclinical Cell and Gene Therapy, a section of the journal Frontiers in Bioengineering and Biotechnology

Received: 26 October 2020 Accepted: 07 January 2021

Published: 12 February 2021

Citation: Kouroupis D and Correa D (2021) Increased Mesenchymal Stem Cell

Functionalization

in Three-Dimensional Manufacturing Settings for Enhanced Therapeutic Applications.

Front. Bioeng. Biotechnol. 9:621748. doi: 10.3389/fbioe.2021.621748

\section{Dimitrios Kouroupis ${ }^{1,2 *}$ and Diego Correa ${ }^{1,2}$}

${ }^{1}$ Department of Orthopedics, UHealth Sports Medicine Institute, University of Miami, Miller School of Medicine, Miami, FL, United States, ${ }^{2}$ Diabetes Research Institute \& Cell Transplantation Center, University of Miami, Miller School of Medicine, Miami, FL, United States

Mesenchymal stem/stromal cell (MSC) exist within their in vivo niches as part of heterogeneous cell populations, exhibiting variable stemness potential and supportive functionalities. Conventional extensive 2D in vitro MSC expansion, aimed at obtaining clinically relevant therapeutic cell numbers, results in detrimental effects on both cellular characteristics (e.g., phenotypic changes and senescence) and functions (e.g., differentiation capacity and immunomodulatory effects). These deleterious effects, added to the inherent inter-donor variability, negatively affect the standardization and reproducibility of MSC therapeutic potential. The resulting manufacturing challenges that drive the qualitative variability of MSC-based products is evident in various clinical trials where MSC therapeutic efficacy is moderate or, in some cases, totally insufficient. To circumvent these limitations, various in vitro/ex vivo techniques have been applied to manufacturing protocols to induce specific features, attributes, and functions in expanding cells. Exposure to inflammatory cues (cell priming) is one of them, however, with untoward effects such as transient expression of HLA-DR preventing allogeneic therapeutic schemes. MSC functionalization can be also achieved by in vitro 3D culturing techniques, in an effort to more closely recapitulate the in vivo MSC niche. The resulting spheroid structures provide spatial cell organization with increased cell-cell interactions, stable, or even enhanced phenotypic profiles, and increased trophic and immunomodulatory functionalities. In that context, MSC 3D spheroids have shown enhanced "medicinal signaling" activities and increased homing and survival capacities upon transplantation in vivo. Importantly, MSC spheroids have been applied in various preclinical animal models including wound healing, bone and osteochondral defects, and cardiovascular diseases showing safety and efficacy in vivo. Therefore, the incorporation of 3D MSC culturing approach into cell-based therapy would significantly 
impact the field, as more reproducible clinical outcomes may be achieved without requiring ex vivo stimulatory regimes. In the present review, we discuss the MSC functionalization in $3 \mathrm{D}$ settings and how this strategy can contribute to an improved MSC-based product for safer and more effective therapeutic applications.

Keywords: MSC functionalization, MSC spheroids, MSC therapeutic properties, MSC anti-inflammatory properties, mesenchymal stem cell manufacturing

\section{MSC THERAPEUTIC PROPERTIES IN CONVENTIONAL 2D CULTURES}

Mesenchymal stem/stromal cells (MSC) are non-hematopoietic cells first isolated from the bone marrow tissue by Friedenstein et al. (1974), and thereafter from various other connective tissues and biological fluids including fat pad (Dragoo et al., 2003), adipose (Zuk et al., 2002), synovium (De Bari et al., 2001), synovial fluid (Jones et al., 2008), and umbilical cord (Weiss and Troyer, 2006). The perceived advantage of MSC as cell therapy is associated with their ease of isolation and high proliferative capacity while retaining their stemness in vitro, but most importantly their paracrine immunomodulatory and trophic (i.e., angiogenic, anti-fibrotic, anti-apoptotic, and mitogenic) actions in vivo. On this basis, MSC "medicinal signaling" activities (Caplan, 2017) exploit their environmental sensory capacity and by secretion of modulatory mediators induce the restoration of the distorted local homeostasis of the target tissue. The immunomodulatory effects of MSC are mediated by secreted bioactive molecules (i.e., IDO, PGE2, TGF $\beta$, IGF, and IL-10), and by cell-cell contact affecting both innate and adaptive immunity (Waterman et al., 2010; Caplan and Correa, 2011; Singer and Caplan, 2011; Bernardo and Fibbe, 2013; Krampera et al., 2013; Uccelli and Rosbo, 2015; Kouroupis et al., 2017, 2018). The trophic effects are mediated by several bioactive molecules resulting in anti-apoptotic [VEGF, HGF, IGF-I, stanniocalcin-1 (STC-1), TGF- $\beta$, and GM-CSF] and mitotic (SCF, LIF, M-CSF, SDF-1, and angiopoietin-1) effects on tissue-intrinsic progenitors (da Silva Meirelles et al., 2009). Most importantly, MSC support the new vessel formation not only by functioning as pericytes and stabilizing newly formed vasculature (Sorrell et al., 2009) but also by secreting ECM molecules and angiogenic factors (VEGF, IGF1, PIGF, MCP-1, bFGF, and IL-6) (da Silva Meirelles et al., 2009).

As reviewed in Kouroupis et al. (2017), MSC therapeutic usage in vivo in both autologous and allogeneic settings is safe due to their immunoevasive characteristics, and therefore, even multiple infusions of allogeneic MSC do not elicit a strong immune response that can lead to rejection progression (Koç et al., 2002; Aggarwal and Pittenger, 2005; Ringden et al., 2006; Le Blanc et al., 2008; Pittenger et al., 2019). Over the past 30 years, the safety profile of MSC has been clearly demonstrated in clinical trials to treat multiple clinical indications, with efficacy starting to produce encouraging results in some of them. To date, more than 10,000 patients have been treated as part of clinical trials, with 188 phase 1 or phase 2 trials completed and 10 trials advanced to phase $3 .^{1}$ However, to obtain clinically

${ }^{1}$ www.clinicaltrials.gov relevant cell numbers, therapeutic protocols usually require MSC extensive in vitro $2 \mathrm{D}$ expansion resulting in MSC products with limited stem cell potency and, as a result in some cases, only moderate or inconsistent effectiveness to treat various clinical indications. Also, according to previous studies, MSC isolated from different tissue sources demonstrate similar, but not identical, functional capacity (Guilak et al., 2010; Moretti et al., 2010; Hass et al., 2011). Efficacy and reproducibility of MSC therapies are not only affected by the composition of the cell preparation but also by the functionality of the infused MSC to consistently home and engraft within dysregulated tissues, and subsequently to predictably exert their therapeutic effects by inducing and/or modifying specific host responses. To circumvent these limitations, various in vitro/ex vivo techniques have been applied to manufacturing protocols to induce specific features, attributes, and functions in expanding cells. On this basis, MSC functionalization can be achieved by in vitro $3 \mathrm{D}$ culturing techniques, in an effort to more closely recapitulate the in vivo 3D MSC niche and therefore preserve or enhance cellular phenotypes that result in improved in vivo therapeutics.

\section{MSC SPHEROID FORMATION AND STRUCTURE}

Adult MSC possesses a remarkable ability to coalesce and assemble in tri-dimensional (3D) structures, reminiscent of their innate aggregation as limb cell precursors in the mesenchymal condensation during early skeletogenesis. In that context, 3D organoid formation in vitro closely recapitulates the in vivo MSC niche by providing spatial cell organization with increased cell-cell interactions.

According to the differential adhesion hypothesis that was first introduced in the 1960s, the cell movement and cell aggregation phenomena present in self-assembly processes are driven by differential cadherin expression levels and guided by the reduction of adhesive-free energy as cells tend to maximize their mutual binding (Foty and Steinberg, 2005). In general, cell aggregation and subsequent multicellular spheroid formation processes involve three phases (Figure 1A). Initially, cells form loose aggregates via the tight binding of extracellular matrix arginine-glycine-aspartate (RGD) motifs with membrane-bound integrin. As a result of the increased cellcell interactions, cadherin gene expression levels are upregulated, whereas cadherin protein is accumulated on the cell membrane. During the later phase, homophilic cadherin-to-cadherin binding induce the formation of compact cell spheroids from cell aggregates. The extracellular matrix proteins and cadherin type 


\section{A}

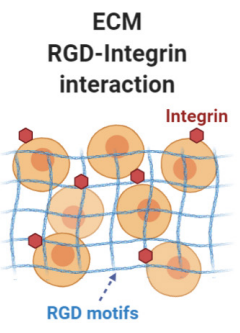

Cell aggregation

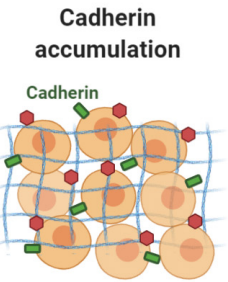

Cadherin-to-Cadherin

homophilic binding
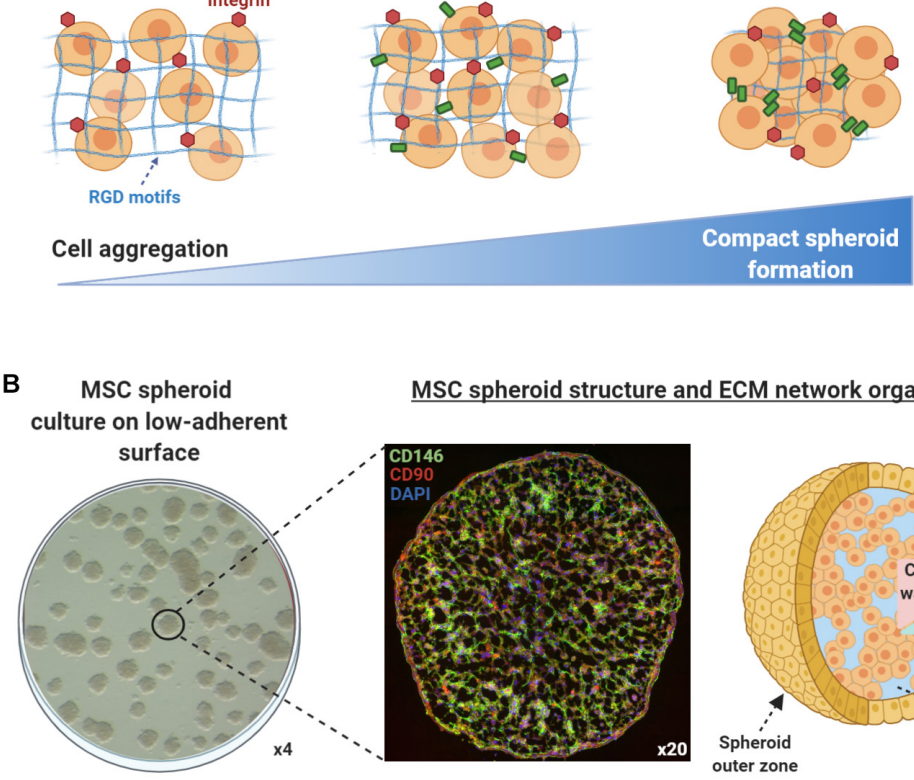

Compact spheroid formation

FIGURE 1 | Mesenchymal stem/stromal cell (MSC) spheroids formation process and structure. (A) Cell aggregation and spheroid formation involving three phases. Initially, cells form loose aggregates via the tight binding of extracellular matrix arginine-glycine-aspartate (RGD) motifs with membrane-bound integrin. Due to increased cell-cell interactions, cadherin gene expression levels are upregulated and cadherin protein is accumulated on the cell membrane. In the later phase, homophilic cadherin-to-cadherin binding induce the formation of compact cell spheroids from cell aggregates. (B) Methylcellulose-based technique can be used to generate viable MSC spheroids on low-attachment gas-permeable plates (left panel). Generated MSC spheroids show stable immunophenotypic profile by expressing high levels of the pericytic marker CD146 (green) and MSC-related marker CD90 (red) (middle panel) (unpublished data). Structurally, based on their size and abundance of nutrients and oxygen in vitro, MSC spheroids can be divided into zones (outer and inner). The nutrients, oxygen, and waste concentration gradients within the spheroids should be always taken into consideration when selecting the optimal technique to generate spheroids in vitro in order to achieve increased spheroid functionality in in vivo settings (right panel).

and concentration are variable between different cell types, whereas other intercellular proteins such as connexin, pannexins, and actin cytoskeleton filaments play crucial roles in cell-cell interactions and subsequent multicellular cell spheroid formation (reviewed in Cui et al., 2017). Structurally, based on their size and abundance of nutrients and oxygen in vitro, most multicellular spheroids can be divided into three zones (Mueller-Klieser, 1984; Alvarez-Pérez et al., 2005; Curcio et al., 2007; Figure 1B). The outer asynchronously proliferative zone contains cells with intact nuclei that are proliferative with active metabolism. The intermediate zone contains cells with shrunk nuclei that are in quiescent state possessing minimum metabolic activities. Usually depending on the spheroid size, the inner necrotic zone contains cells with disintegrated nuclei that are senescent/apoptotic due to limited nutrients and oxygen influx (hypoxia) in the spheroid core. The inner necrotic zone is formed as the diffusion limitation of most molecules in spheroids is $150-200 \mu \mathrm{m}$, and as a result, metabolic wastes are gradually accumulating within the spheroid core. Additionally, Curcio et al. (2007) indicated that aggregates of $200-\mu \mathrm{m}$ diameter or greater show severe oxygen limitation in the most part of their dimensions, and Alvarez-Pérez et al. (2005) related drastic intra-spheroidal $\mathrm{pH}$ alterations to spheroid size, with spheroids of $600-\mu \mathrm{m}$ diameter or greater showing acidic necrotic core. Based on these findings, a three-part spheroid zonation is highly dependent on cell aggregation size and microenvironment conditions, whereas a $200-\mu \mathrm{m}$ diameter can be putatively considered a reliable size threshold for limited/diminished inner necrotic core zone formation. Therefore, the nutrients, oxygen, and waste concentration gradients within the spheroids should be always taken into consideration when selecting the optimal technique to generate spheroids in vitro in order to achieve increased spheroid functionality in in vivo settings.

The organization of MSC in 3D spheroids result in altered cell morphology, cytoskeleton rearrangement, and polarization due to the cell-cell and cell-extracellular matrix interactions within the spheroid structure. Additionally, 3D cultures account for the established reduction in size of individual MSC (about $0.25-0.5$ the volume of an average 2D cultured cell) (Bartosh et al., 2010). Specifically, studies showed that individual MSC strain is increased within the spheroid structure and equally dispersed in all cell dimensions (a Young's Modulus of approximately 60 Pascal), whereas overall MSC tension is greater in the outer zone compared with the inner zone of spheroids. These tension differences affect MSC morphology and polarization resulting in a more flattened morphology and high integrin expression 
for outer zone MSC and a more irregular morphology with high cadherin expression for the inner zone MSC (Baraniak et al., 2012; Sart et al., 2014). On this basis, Lee et al. (2012) indicated E-cadherin as the main calcium-dependent adhesion molecule that plays a crucial role in MSC spheroid formation in vitro. During spheroid formation, E-cadherin activation and cell-cell interactions regulate the proliferative and paracrine activity of MSC via the ERK/AKT signaling pathway (Lee et al., 2012). Importantly, studies showed that cadherins, and especially $\mathrm{N}$-cadherin and $\mathrm{OB}$-cadherin, are both affecting the proliferation, migration, and differentiation potential of 2D MSC cultures (Theisen et al., 2007; Xu et al., 2013). Of note, cadherin levels may be important in mediating MSC anti-inflammatory actions as reports indicated that they are crucial in the response of synovial fibroblasts to inflammation (Agarwal and Brenner, 2006; Chang et al., 2011). To this end, engineered cadherin surfaces and engineered surface microtopology have been generated to control differentiation, and cell-to-cell adhesion and signaling of $2 \mathrm{D}$ cultured MSC in vitro (reviewed in Alimperti and Andreadis, 2015). However, the inherent increased cadherin levels upon MSC spheroid formation can be directly related to increased MSC spheroid functionality in vitro and in vivo, offering an advantage over 2D MSC cultures.

Interestingly, studies showed that mild hypoxia present within the inner zones of MSC spheroids positively affect MSC survival and secretory capacity. Moreover, spheroid hypoxic microenvironment upregulate the expression of hypoxiaadaptive molecules (such as CXCL12 and HIF-1 $\alpha$ ), inhibit MSC apoptosis, and increase the secretion of angiogenic and antiapoptotic molecules including HGF, VEGF, and FGF-2 compared to 2D MSC cultures (Bhang et al., 2011). Specifically, studies showed that MSC spheroids embedded in fibrin gel secrete up to 100-fold more VEGF compared with dissociated MSC in fibrin gel (Murphy et al., 2014). Except these molecules, the angiogenic trophic enhancement is produced via the upregulation of other key angiogenic factors such as angiogenin (ANG) and angiopoietin 2 (ANGPT-2; Potapova et al., 2007; Potapova et al., 2008; Yeh et al., 2014). However, Murphy et al. (2017) reported that even though cellular metabolism decreased significantly with higher cell numbers and resultant spheroid sizes, oxygen tension show a gradient that vary less than $10 \%$ from the outer zone to the inner core even for spheroids with diameters up to $353 \pm 18 \mu \mathrm{m}$. This indicates that increased MSC functionality within the spheroid is not oxygen gradient driven but due to increased ECM production and autocrine signaling. Overall, the advantages and disadvantages of MSC functionalization in $3 \mathrm{D}$ spheroids are described in Table 1.

\section{METHODS AND BIOMATERIALS USED TO GENERATE MSC SPHEROIDS EX VIVO}

Lately, standardization of MSC manufacturing has been extensively evaluated in order to translate in vitro and in vivo preclinical research into safe and effective therapeutic products. Toward this goal, the large-scale clinical-grade generation of
TABLE 1 | Advantages and disadvantages of MSC functionalization in 3D spheroids.

\begin{tabular}{ll}
\hline Advantages & Disadvantages \\
\hline - Increased stability of MSC & - Size variability depending on the \\
immunophenotypic and molecular & technique used to generate spheroids \\
profiles & - Depending on spheroid size, nutrients, \\
- Enhanced stemness features and & oxygen, and waste concentration \\
differentiation potential & gradients within the spheroids \\
- Upon infusion, enhanced survival and & - Depending on spheroid size, necrotic \\
homing in vivo & spheroid core formation \\
- Enhanced secretory profile exerting & - Depending on the clinical needs, \\
mitogenic, anti-apoptotic, & development of reproducible, simple \\
angiogenic, anti-fibrotic and & and cost-effective techniques are \\
anti-inflammatory properties & needed for large-scale production of \\
& MSC spheroids \\
\hline
\end{tabular}

MSC spheroids possessing enhanced functionality in vivo is an imminent need for various therapeutic applications. To date, various methods have been used to generate MSC spheroids including the "classic" hanging drop technique and other improved methods such as the application of low-adhesive substrates, the membrane-based aggregation, and the forced aggregation techniques (reviewed in Petrenko et al., 2017).

\section{SCAFFOLD-FREE MESENCHYMAL STEM/STROMAL CELL SPHEROID CULTURE PLATFORMS}

Mesenchymal stem/stromal cell spheroid culture platforms are usually trivial, rapid, and low-cost methods to generate spheroids in a non- or low-adherent environment that allows the selforganization of cells into suspended spheroids (Figures 2AD). In the hanging drop technique, MSCs are aggregated by gravitational force but due to the absence of direct contact with solid surfaces, the composition of ECM proteins is the main factor for the regulation of spheroid microenvironment (Foty, 2011). Therefore, the hanging drop technique can generate MSC spheroids of controlled size and number; however, its main limitation is the laborious preparation of the $3 \mathrm{D}$ cultures that significantly limits the large-scale production of spheroids for in vivo applications. Using the hanging drop technique, Bartosh et al. indicated a 100-fold upregulation of antiinflammatory (TSG-6) and anti-tumorigenic (IL-24 and TRAIL) genes compared to 2D MSC cultures (Bartosh et al., 2010). In addition, the hanging drop technique results in higher expression of stemness markers Oct4, Sox2, and Nanog in MSC spheroids compared to 2D MSC cultures (Lou et al., 2016). Forced aggregation technique (or pellet culture) is also used to generate scaffold-free MSC aggregates by gravitational force that are further induced toward 3D differentiation protocols such as high-density MSC chondrogenic pellet culture (Mackay et al., 1998). A less laborious and more standardized technique is the use of low-attachment surfaces. Similar to the hanging drop technique, spontaneously secreted ECM proteins are regulating the spheroid microenvironment, however, generated spheroids show increased variability in size and morphology 
A

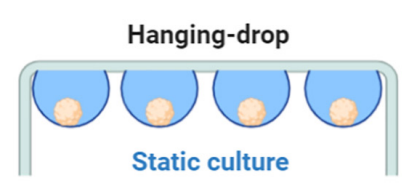

D

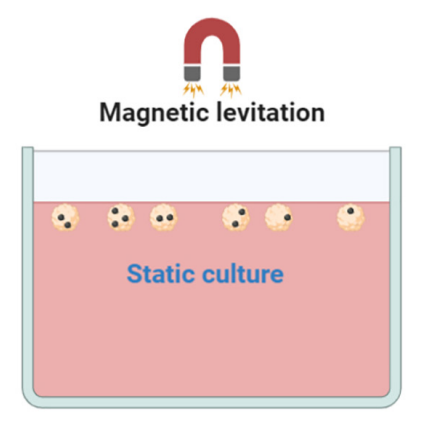

B

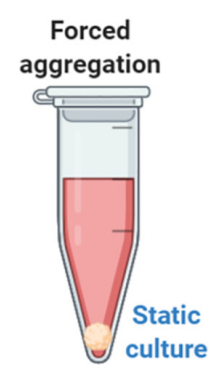

$\mathrm{E}$

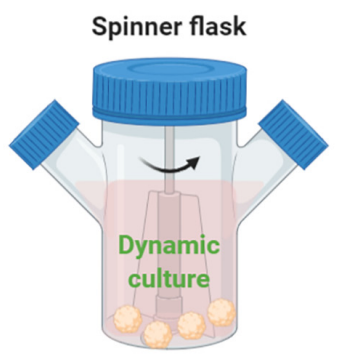

C

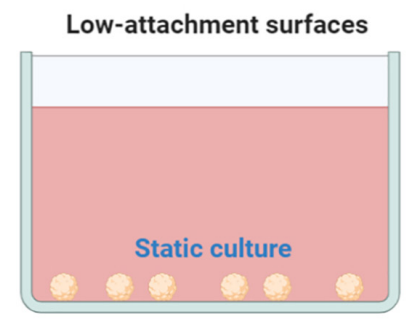

$\mathbf{F}$

FIGURE 2 | Methods used to generate MSC spheroids ex vivo. (A) In hanging drop technique, MSCs are aggregated by gravitational force. (B) Forced aggregation technique (or pellet culture) is used to generate MSC aggregates by gravitational force that are further induced toward 3D differentiation protocols.

(C) Low-attachment surfaces allow the self-organization of cells into suspended spheroids. (D) Magnetic levitation diminish gravitational force, promotes cell-cell contact and induces cell aggregation in vitro. (E) In a spinner flask bioreactor system, cells are continuously mixed by stirring. (F) Rotating wall vessel technique simulates microgravity by constant circular rotation and, therefore, cells are continuously in suspension. Both dynamic culturing techniques (spinner flask and rotating wall vessel) form viable compact MSC spheroids but with altered cell size, altered phenotypic and molecular profiles, and enhanced differentiation potential compared to 2D conventional MSC cultures.

(Redondo-Castro et al., 2018b). Interestingly, studies showed that MSC spheroids generated on low-attachment surfaces secreted more hypoxia-induced angiogenic cytokines including VEGF, SDF, and HGF, whereas phosphorylation of Akt cell survival signaling was higher and the expression of pro-apoptotic molecules lower in MSC spheroids compared with 2D MSC cultures (Lee et al., 2016). Magnetic levitation can be used to generate MSC spheroids as by diminishing gravitational force, it promotes cell-cell contact and induces cell aggregation in vitro. In detail, cells are mixed with magnetic particles in culture, and cells incorporated with them can levitate due to exogenously applied magnetic field. Although preliminary studies show spheroid formation reproducibility and stable MSC spheroid phenotype, others have reported that abnormal gravity induces classic apoptotic alterations such as cell size reduction and cell membrane blebbing, reduced cell viability, nuclear chromatin condensation and margination, and increased caspase-3/7 activity (Meng et al., 2011).

Except the static techniques, various dynamic approaches have been investigated to generate MSC spheroids including spinner flask culture and rotating wall vessel techniques (Figures 2E,F). Spinner culture technique is based on a spinner flask bioreactor system where cells are continuously mixed by stirring, whereas rotating wall vessel technique simulates microgravity by constant circular rotation where cells are continuously in suspension. In a comparative study between dynamic and 2D MSC cultures, Frith et al. indicated that both spinner and rotating wall vessel dynamic cultures can form viable compact MSC spheroids showing altered cell size, altered phenotypic and molecular profiles, and enhanced osteogenic and adipogenic differentiation potential (Frith et al., 2010). Further studies showed that rotating wall vessel microgravity dramatically affect the molecular profile of MSC spheroids by upregulating genes related to adipogenic and downregulating genes related to osteogenic and chondrogenic differentiation potentials (Sheyn et al., 2010). MSC spheroid culturing in microgravity conditions results in reduced osteogenic differentiation due to decreased Collagen I gene expression and subsequent Collagen I/integrin signaling pathway activation (Meyers et al., 2004). Also, microgravity disrupts F-actin stress fibers, increase intracellular lipid accumulation, and significantly reduces RhoA activity (Meyers et al., 2005). Interestingly, others indicated that microgravity has a synergistic effect with chemical induction in stimulation of chondrogenesis mediated by p38 MAPK activation (Yu et al., 2011).

The abovementioned advantages of MSC spheroids over 2D MSC cultures make them a great candidate as building blocks for 3D bioprinting. For the large-scale manufacturing of spheroid-based tissue complexes in vitro, various 3D bioprinting techniques have been reported including extrusionbased bioprinting (Jakab et al., 2008; Mironov et al., 2009; Bulanova et al., 2017; Mekhileri et al., 2018), droplet-based bioprinting (Gutzweiler et al., 2017), Kenzan (Moldovan et al., 2017), and biogripper (Blakely et al., 2015; Ip et al., 2016) approaches. Studies showed that homogeneous MSC-derived 
cartilage spheroids with a mean diameter of $116 \pm 2.8 \mu \mathrm{m}$ can be assembled using extrusion-based bioprinting into viable cartilage constructs with stable phenotype (De Moor et al., 2020). Also, MSC-derived adipose spheroids bioprinted into a microtissue showed multilocular microvacuoles and successful differentiation toward mature adipocytes (Colle et al., 2020). However, existing 3D bioprinting techniques involve several limitations related to substantial damage to biological, structural, and mechanical spheroid properties. Recently, Ayan et al. (2020) proposed aspiration-assisted bioprinting as a novel approach for MSC spheroid assembly that causes minimal cellular damage and precisely bioprint a wide range of spheroid dimensions (ranging from 80 to $800 \mu \mathrm{m}$ ). On this basis, authors demonstrated the patterning of angiogenic sprouting spheroids and self-assembly of osteogenic spheroids. Further advancements into bioprinting field would benefit the generation of various types of MSC spheroid-derived microtissues in vitro.

\section{SCAFFOLD-BASED MESENCHYMAL STEM/STROMAL CELL SPHEROID CULTURE PLATFORMS}

In addition to the scaffold-free culture platforms, various scaffold-based MSC spheroid generation approaches have been proposed using both natural and synthetic biomaterials. As mentioned before, MSC spheroids can benefit the in vivo microenvironment primarily by their immunomodulatory and trophic actions, and secondarily (if any) by their direct differentiation toward specialized cells. The latter supports the notion that MSC spheroids should maintain their integrity in order to achieve effective cell replacement in vivo as biodegradation is a key factor in tissue engineering. Therefore, depending on the therapeutic application mode, biomaterial selection except from biological factors (cell adhesion, biocompatibility, etc.) should take into consideration physic-chemical (porosity to support nutrients/oxygen influx, biodegradation, etc.) parameters (Nikolova and Chavali, 2019). On this basis, even though scaffold's topography allows seeded MSC to form a microstructured matrix within the 3D spheroid microenvironment, depending on the treated tissue's nature, scaffold biodegradation rate should be controlled accordingly by the incorporation of chemical components that trigger gradual hydroytic degradation. However, to date, no specific studies have been performed to define if long-term maintenance of MSC spheroid structure is crucial for its therapeutic use.

Scaffold-based culture platforms using natural polymers such as agar/agarose, chitosan, and collagen can promote spheroid formation. Agar/agarose non-adherent surfaces have been used to promote MSC aggregation and spheroid formation in vitro (Vorwald et al., 2018). Specifically, chitosan-based substrates result in a more complex spheroid microenvironment compared to scaffold-free methods as the carbohydrate structure of chitosan is similar to the glycosaminoglycans in the ECM (Cui et al., 2017). Chitosan is a polycationic natural biocompatible polysaccharide, whereas the degree of its deacetylation can modulate the cell adhesion and spheroid formation capacity in vitro. On this basis, highly deacetylated chitosan substrate supports strongly the attachment and proliferation of fibroblasts (Seda Tiğlı et al., 2007). Interestingly, Yeh et al. showed that MSC spheroid culturing on chitosan membranes results in increased intracellular calcium levels, whereas the calcium binding capacity of chitosan affect the cell-substrate and cell-cell interactions within the MSC spheroid. As a result, the chitosan-cultured MSC spheroids show significantly upregulated expression of calcium-, cell adhesion/ migration-, and anti-inflammatory-associated genes compared to 2D MSC on tissue culture polystyrene plates (Yeh et al., 2012, 2014). Hsu and Huang showed that Wnt signaling is not only distinct in MSC spheroids compared to 2D MSC cultures but also substrate dependent. MSC spheroids derived on chitosan-activated Wnt $3 \alpha$-mediated canonical Wnt signaling is prone to osteogenesis, whereas MSC spheroids derived on hyaluronan-grafted chitosan activated Wnt $5 \alpha$-mediated non-canonical Wnt signaling that is prone to chondrogenesis (Hsu and Huang, 2013). On this basis, Huang et al. (2011) showed that MSC spheroids generated on chitosan and chitosan-hyaluronan substrates preserve the expression of stemness markers Oct4, Sox2, and Nanog, and increase their chondrogenic differentiation capacity. As autophagy is an important mechanism promoting cell survival, a study showed that MSC spheroids derived on chitosan respond to environmental stress $\left(\mathrm{H}_{2} \mathrm{O}_{2}\right.$ treatment $)$ by upregulating autophagy-related markers in a calcium-dependent manner (Yang et al., 2015). This effect is important as it may increase the MSC spheroid survival and therapeutic efficacy in in vivo settings. Interestingly, nanomagnetically levitated MSCs cultured as spheroids within type I collagen gels preserve their quiescent phenotype indicated by the expression of STRO-1 and Nestin, whereas in response to co-culture wounding, they are capable of migrating to the wound site and differentiate accordingly (Lewis et al., 2016).

Polymers and chemically modified polymers have been extensively investigated for the development of novel biomaterials with good physic-chemical properties and biocompatibility. On this basis, MSC spheroid generation has been performed on various synthesized polymer substrates such as polycaprolactone, micropatterned poly(ethylene glycol), poly(L-glutamic acid)/chitosan, and methylcellulose. In one study, Messina et al. (2017) showed that fibroblast, myoblast, and neural cell spheroids on polymeric membranes possess high biological activity in terms of oxygen uptake, whereas they undergo faster fusion and maturation on polycaprolactone than on agarose substrates. Also, Wang W. et al. (2009) showed improved adipogenic and osteogenic differentiation capacity of MSC spheroids generated on micropatterned poly(ethylene glycol) substrates. Microarray analysis indicated not only the upregulation of genes related to adipogenesis and osteogenesis but also the downregulation of genes related to MSC stemness such as the mesoderm-specific transcript (MEST) and the mesenchymal stem cell specific marker (THY1) (Wang W. et al., 2009). Similarly, Zhang et al. (2015) indicated that MSC spheroids generated on poly(L-glutamic acid)/chitosan substrate show increased chondrogenic differentiation capacity by increased GAGs and COLII, and decreased COLI deposition during in vitro chondrogenic induction. 
Methylcellulose, an ether derivative of cellulose, which is synthesized by the replacement of hydrogen atoms from hydroxy groups with methyl groups, has been recently used to generate successfully MSC spheroids in vitro. Deynoux et al. (2020) showed that methylcellulose allows MSC spheroid formation within $24 \mathrm{~h}$, which tends to shrink in size partially due to the balance between proliferation and cell death triggered by hypoxia and oxidative stress up to 3 weeks in vitro. Similar to methylcellulose-based technique published by Markou et al. (2020), we have generated successfully viable MSC spheroids in a gas-permeable plate system that possess stable phenotypic and molecular profiles, and increased functionality both in vitro and in vivo (Kouroupis et al., 2021). The usage of this system is aimed to ensure uniform oxygenation throughout the MSC spheroid culture, as it is based on previous reports demonstrating that in gas-permeable plates 3D cell structures efficiently receive air from both the top (after diffusion through the medium) and the bottom (after diffusion across permeable membrane) of the culture (Fraker et al., 2007, 2013; Cechin et al., 2014). These reports show that MSC spheroid generation on synthesized substrates can dramatically affect their stemness and multipotential differentiation capacities in vitro.

\section{CULTURE MEDIUM EFFECTS ON MESENCHYMAL STEM/STROMAL CELL SPHEROIDS}

With the exception of the scaffold-free or scaffold-based culture platforms, reports showed that culture medium composition strongly affect the spheroid formation progression and MSC spheroid functionality in vitro. To date, most studies use fetal bovine serum (FBS)-based media to generate spheroids in vitro. However, safety concerns have been raised regarding FBS usage for the manufacturing of MSC products for clinical applications, most of them related to prion exposure risk, toxicological risk, and immunological risk (Mendicino et al., 2014; Karnieli et al., 2017). Regulatory-complaint xeno-free media such as chemically defined formulations and human platelet lysate (hPL) are promising alternatives to generate clinically relevant cell numbers and to preserve or even enhance the MSC functionality in vitro prior to their in vivo application (Doucet et al., 2005; Centeno et al., 2008; Jung et al., 2010; Kouroupis et al., 2020a,b). On this basis, Ylostalo et al. (2014) showed that MSCs cannot condense into tight spheroids when cultured in several commercial stem cell media and only chemically defined formulation supplemented with human serum albumin (HSA) can result in compact MSC spheroids with high viability and enhanced anti-inflammatory secretory profile. Importantly, MSC spheroids generated with HAS supplementation show increased anti-inflammatory capacity when co-cultured with lipopolysaccharide-stimulated macrophages in vitro (Ylostalo et al., 2014). In contrast, another study indicated that MSC spheroids generated in FBS-based medium show low or no proliferation but increased paracrine secretory profile (PGE2 and IDO), whereas MSC spheroids generated in xeno-free medium show significant proliferative capacity but low paracrine secretory profile (Zimmermann and McDevitt, 2014).

Overall, further investigations have to be performed in order to optimize the in vitro culturing conditions for the standardization and reproducibility of MSC spheroid therapeutic potential. Most importantly, challenges still exist related to the generation of clinically relevant cell numbers in 3D cultures and the qualitative assessment of the generated MSC spheroids using conventional methods. Specifically, the less laborious dynamic approaches, such as the spinner flask culture and the rotating wall vessel techniques, offer a viable solution to generate large MSC spheroid numbers; however, novel bioreactor systems are needed to additionally monitor and control all culture environmental variables (temperature, gas exchange, $\mathrm{pH}$, and metabolite levels) (de Bournonville et al., 2019). Similar to 2D MSC cultures, qualitative evaluation of MSC spheroids requires their phenotypic protein profiling using fluorescent microscopy and flow cytometry methods. Fluorescent imaging is often laborious for xyz images and represent only a fraction of MSC spheroid cultures, whereas flow cytometry requires the enzymatic/mechanical dissociation of the spheroids to a single cell, usually disrupting important sensitive phenotypic attributes (CD146 immunomodulationrelated marker). Furthermore, comparative preclinical studies are needed to evaluate how different MSC spheroid generation platforms in vitro are affecting the therapeutic outcomes upon their implantation or infusion in vivo.

\section{ANTI-INFLAMMATORY PROPERTIES OF MESENCHYMAL STEM/STROMAL CELL SPHEROIDS}

In MSC spheroid settings, their enhanced anti-inflammatory effects have been mainly attributed to high expression of TGF- $\beta 1$, IL-6, TSG-6, stanniocalcin (STC-1), and PGE-2 antiinflammatory molecules (Bartosh et al., 2010; Ylöstalo et al., 2012; Zimmermann and McDevitt, 2014; Figure 3). Specifically, Bartosh et al. showed that BM-derived MSC spheroid increased secretion of anti-inflammatory TSG-6 and STC-1 results in reduced TNF $\alpha$ expression and secretion by LPS-stimulated macrophages in MSC spheroid/macrophages co-cultures in vitro. In a mouse zymosan-induced peritonitis model, intraperitoneal injection of $1.5 \times 10^{6} \mathrm{BM}$-derived MSC spheroids for a 6-h time-frame resulted in decreased protein content and volume of the lavage fluid, neutrophil activity, and decreased levels of TNF $\alpha$, IL-1 $\beta$, CXCL2/MIP-2, and PGE2. Also, MSC spheroid injection significantly decreased the serum levels of plasmin activity, an inflammation-related protease that is inhibited by secreted TSG-6 (Bartosh et al., 2010). Importantly, in vitro studies showed that BM-derived MSC spheroid conditioned medium affect LPS-stimulated macrophages not only by inhibiting the secretion of pro-inflammatory cytokines TNF $\alpha$, CXCL2, IL-6, IL12-p40, and IL-23 but also by increasing the secretion of antiinflammatory cytokines IL-10 and IL1-Ra and the expression of M2-polarization CD206 marker. The main anti-inflammatory molecule secreted in the conditioned medium was PGE2, whereas 


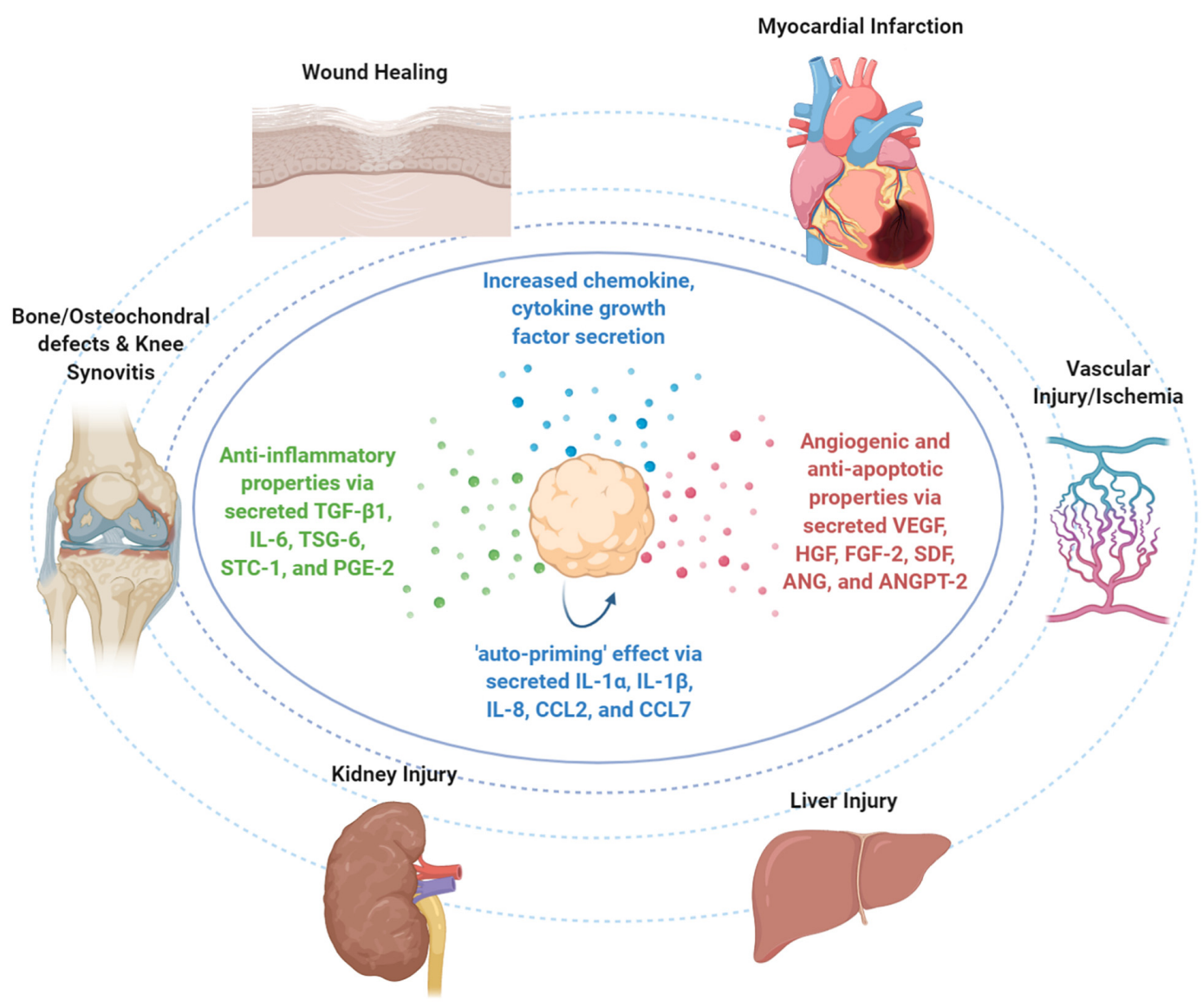

FIGURE 3 | Therapeutic properties of MSC spheroids in vivo. Upon infusion in vivo, MSC spheroid "medicinal signaling" activities are exerted by the paracrine secretion of modulatory mediators that possess immunomodulatory and trophic (i.e., angiogenic, anti-fibrotic, anti-apoptotic, and mitogenic) actions. MSC spheroids have been safely and effectively applied in various preclinical animal models for the treatment of skin wounds, myocardial infarction, vascular injury/ischemia, liver injury, kidney injury, bone and osteochondral defects, and knee synovitis.

its production is dependent on caspase activity and NFkB activation in MSC spheroids (Ylöstalo et al., 2012).

Upon MSC homing to the target site and depending on the molecular composition of the local microenvironment, they exhibit a therapeutic responsive polarization into either anti-inflammatory (MSC-2) or pro-inflammatory (MSC1) phenotypes. Interestingly, studies showed that except for the abovementioned secreted molecules with antiinflammatory effects, MSC spheroids increase the secretion of pro-inflammatory cytokines (including IL- $1 \alpha$, IL-1 $\beta$, and IL-8) and chemokines (including CCL2 and CCL7) (Potapova et al., 2007; Bartosh et al., 2010, 2013; Yeh et al., 2014) that contribute in the inflammatory cell recruitment locally and putatively in the overall inflammatory response of the host. However, Bartosh et al. showed that BM-derived MSC assembly into MSC spheroids triggers the caspase-dependent IL-1 signaling and activates the expression of IL- 1 in an autocrine secretion manner, resulting in an "auto-priming" effect (Figure 3). In MSC spheroids, the increased PGE2 secretion was related to activation of both caspase-dependent IL-1 and Notch signaling pathways, whereas TSG-6 and STC-1 secretion was related only to caspase-dependent IL-1 signaling activation (Bartosh et al., 2010). Collectively, MSC priming by paracrine and/or autocrine pro-inflammatory modes is a prerequisite in order to acquire their anti-inflammatory MSC2 phenotype and exert strong anti-inflammatory effects in vivo.

As reviewed in Kouroupis et al. (2018), several studies indicate that activation of specific Toll-like receptors (TLRs) in MSC in vitro prior to infusion in vivo has a profound effect on MSC functionalization toward immunomodulatory phenotype. However, Redondo-Castro et al. (2018a) reported that IL-1 stimulation of BM-derived MSC spheroids resulted in significantly increased expression of IL1-Ra, VEGF, and G-CSF molecules without anti-inflammatory effects on LPStreated microglial cells in co-cultures. These discrepancies of the data underline the necessity for optimization of the priming methods and culture conditions. Previous studies showed that MSC immunomodulatory factor secretion is strongly affected by the composition of the culture medium (Zimmermann and McDevitt, 2014). In 2D culture settings, BM-derived and adipose-derived MSC cultured with FBS or hPL showed differences in expression of immunomodulatory 
and adhesion molecules, with adipose-derived MSC being more potent functionally in inhibiting T-cell proliferation (Menard et al., 2013). Similarly, in two studies, Kouroupis et al. (2020a; 2020b) indicated that fat pad-derived (IFP) MSCs when cultured in regulatory-compliant conditions in vitro are superior functionally in Substance $\mathrm{P}$ degradation and T-cell proliferation inhibition compared to FBS-grown MSC. In an acute synovitis rat model, IFP-MSC intra-articular injection in vivo reversed more effectively signs of synovitis and IFP fibrosis when they were cultured under regulatorycompliant conditions (Kouroupis et al., 2020a,b). In 3D settings, MSC spheroids cultured in serum and animal component-free chemically defined medium had less secretion of IDO, PGE2, TGF- $\beta 1$, and IL- 6 immunomodulatory factors compared to the typical MSC cultures supplemented with FBS (Zimmermann and McDevitt, 2014). In order to overcome these hurdles, Ylostalo et al. proposed specific protocols to efficiently prime MSCs in 3-D settings and preserve their robust antiinflammatory properties under chemically defined xeno-free conditions (Ylostalo et al., 2017).

Overall, further studies are required to address the effects of pro-inflammatory cytokines and culturing conditions on antiinflammatory properties of MSC spheroids in vitro and in vivo.

\section{THERAPEUTIC PROPERTIES OF MESENCHYMAL STEM/STROMAL CELL SPHEROIDS IN PRECLINICAL ANIMAL MODELS}

With exception to the therapeutic safety that most MSC clinical trials are investigating for various clinical disorders, ${ }^{2}$ crucial factors that affect the therapeutic efficiency are MSC homing to target tissues and subsequent MSC survival in vivo. It cannot be overlooked that initial outcomes from many of such studies revealed that MSC therapies show a significant degree of variability with cases of non-reproducible clinical data. The inconsistent evidence potentially relates not only to intrinsic differences in the cell-based products used but importantly related with their in vivo fate upon implantation or infusion [parameters affecting MSC functionalization in vitro and in vivo are reviewed in Kouroupis et al. (2018)]. On this basis, a pioneering study showed that $5.0 \times 10^{5} \mathrm{BM}$-MSC injected into the left ventricle of uninjured mouse heart can effectively engraft the myocardium; however, only $0.44 \%$ of the MSCs could be identified after 4 days of injection (Toma et al., 2002). In addition, Toma et al. showed that $92 \pm 7 \%$ of intraarterially injected MSC in rats are entrapped in the microvasculature (Toma et al., 2009). Collectively, even though long-term engraftment seems not to be a prerequisite for MSC reparative effects in vivo, their initial homing and survival is a crucial factor affecting the therapeutic outcomes. In that context, 3D spheroid formation in vitro closely recapitulates the in vivo MSC niche by providing spatial cell organization with increased cell-cell interactions that protect MSC viability and intrinsic properties.

${ }^{2} \mathrm{http}: / /$ clinicaltrials.gov
For example, in a mouse model of hind limb ischemia, MSC spheroid transplantation improved its survival compared to MSC suspension, by suppressing a key apoptotic signaling molecule (Bax), while activating anti-apoptotic signaling (BCL-2; Bhang et al., 2012). These positive effects can also be attributed to improved resistance to oxidative stress-induced apoptosis exerted by hypoxia-induced genes (e.g., VEGF-A, HIF-1 ${ }^{\alpha}$, and MnSOD), elevated by the hypoxic conditions at the spheroid core (Potapova et al., 2007; Zhang et al., 2012).

Mesenchymal stem/stromal cell-based spheroids have been applied in various preclinical models including wound healing (Amos et al., 2009; Zhang et al., 2012; Hsu and Hsieh, 2015), bone and osteochondral defects (Ma et al., 2011; Suzuki et al., 2012; Suenaga et al., 2015), knee synovitis (Kouroupis et al., 2021), and cardiovascular diseases (Wang C.-C. et al., 2009; Emmert et al., 2013a) (Figure 3).

\section{WOUND HEALING}

To date, three separate studies applied MSC spheroids for wound healing in a model of diabetic healing impaired (leptin receptordeficient) mice (Amos et al., 2009), in chemotherapy-induced oral mucositis (Zhang et al., 2012), and in a rat skin repair model (Hsu and Hsieh, 2015). In a pioneering study, Amos et al. investigated the applicability of MSC spheroids to treat chronic wounds such as diabetic ulcers, which remain a significant health burden for diabetic patients. In detail, full-thickness dermal wounds (approximately $78.5 \mathrm{~mm}^{2}$ area) were generated in leptin receptor-deficient mice and treated with a total of 350,000 adipose-derived MSC per wound organized in multiple separate spheroids. Interestingly, for a 12 day time-frame, MSC spheroids resulted in significantly greater rate of wound closure compared to wounds treated with MSC suspension. This outcome may be attributed to higher expression of ECM genes (tenascin $C$, Collagen VI 3 , and fibronectin) and higher secretion of soluble factors (HGF, MMP-2, and MMP-14) in MSC spheroid compared to MSC suspension cultures in vitro (Amos et al., 2009). Zhang et al. (2012) intravenously infused $1 \times 10^{6}$ gingiva-derived MSC spheroids or MSC suspension to a 5-fluorouracil-induced oral mycositis mouse model. On day 7 , results indicated that MSC spheroids can reverse body weight loss and promote the regeneration of damaged epithelial lining of the mucositic mouse tongues. Interestingly, authors reported that MSC spheroids are capable of increased homing/engrafting to mucositic tongues due to their enhanced CXCR4 expression and may potentially transdifferentiate into epithelial cells via mesenchymal-epithelial transition in vivo (Zhang et al., 2012). These data indicate the potential use of MSC spheroids to alleviate the oral mucositis side-effect post-chemotherapy in cancer patients. In another rat skin wound healing model, $1 \times 10^{5}$ adipose-derived MSC spheroids or MSC suspension were applied to $15 \mathrm{~mm} \times 15 \mathrm{~mm}$ wounds and covered with hyaluronan gel/chitosan sponge to maintain a moist environment. On day 8, results showed that the MSC spheroid group showed faster wound closure and significantly higher ratio of angiogenesis compared with the MSC suspension group. In vivo tracking of fluorescently labeled MSCs 
showed close localization of MSC spheroids to microvessels, suggesting enhanced angiogenesis through paracrine effects. Moreover, MSC spheroid increased engrafting and angiogenesis effects may be attributed to the high expression of cytokine genes (FGF-1, VEGF, and CCL2) and migration-related genes (CXCR4 and MMP-1) (Hsu and Hsieh, 2015). Collectively, in all cases, MSC spheroids provide better therapeutic efficacy compared with traditional MSC suspension in wound healing.

\section{BONE/OSTEOCHONDRAL DEFECTS AND SYNOVITIS}

Studies showed that bone/osteochondral defects and knee synovitis can be treated by MSC spheroids. In a delicate study, Sekiya's group generated a full-thickness $(5 \mathrm{~mm} \times 5 \mathrm{~mm}$ wide, $1.5 \mathrm{~mm}$ deep) osteochondral defect rabbit model, and defects were treated with different doses of synovium-derived MSC spheroids (containing $2.5 \times 10^{5}-20 \times 10^{6} \mathrm{MSC} /$ defect) (Suzuki et al., 2012). Post-implantation MSC spheroids could attach to the osteochondral defects by surface tension, whereas at 12 weeks, MSC spheroids containing $2.5 \times 10^{6}$ MSC showed the highest safranin-O-positive area ratio and resulted in regenerated cartilage with thickness similar to the neighboring healthy cartilage. Interestingly, authors reported that MSC spheroids with high cell densities result in failed defect repair and fibrous tissue formation possibly due to cell death and nutrient deprivation effects (Suzuki et al., 2012). In a calvarial bone defect $(8 \mathrm{~mm}$ wide) rat model, Suenaga et al. treated the rat defects using three different conditions, $3.0 \times 10^{7}$ BM-MSC spheroids, $\beta$-TCP granules, or BM-MSC spheroids coated with $\beta$-TCP granules. Eight weeks post-implantation, MSC spheroids resulted in full-thickness bone formation with evident vascularization. In contrast, the other two groups had only minimal or non-uniform bone formation at the implanted sites, indicating that $\beta$-TCP restricts the bone regenerative capacity of MSC spheroids (Suenaga et al., 2015). Recently, Yanagihara et al. (2018) treated $4 \mathrm{~mm}$ wide femoral bone defects in rats with $2.4 \times 10^{6}$ Runx2-transfected MSC spheroids or Runx2-transfected MSC suspension embedded in collagen scaffolds. On day 35, MSC spheroids showed faster bone regeneration compared with MSC suspension and nontransfected MSC, whereas enhanced MSC spheroid migration to the defect sites was correlated with higher expression levels of migration-related genes CXCR4 and Integrina2 (Yanagihara et al., 2018). Recently, in a mono-iodoacetate acute synovial/IFP inflammation rat model, Kouroupis et al. intraarticularly injected $5.0 \times 10^{5}$ infrapatellar fat pad MSC (IFP-MSC) spheroids. Twenty-five days post-infusion, IFP-MSC spheroids effectively degraded Substance $\mathrm{P}$ and resolved inflammation and fibrosis of synovial membrane and fat pad tissues in the rat knee. Interestingly, IFP-MSC intraarticular injection not only results in anti-inflammatory and anti-fibrotic effects but also showed strong anabolic/cartilage protective effects. Specifically, in the IFP-MSC spheroid cohort, cartilage integrity was preserved intact up to 28 days (Kouroupis et al., 2021). To conclude, MSC spheroids exert anti-inflammatory/anti-fibrotic effects and are effective for promoting both bone and osteochondral defect regeneration.

\section{MYOCARDIAL INFARCTION}

Intramyocardial transplantation of MSC spheroids in rat (Wang C.-C. et al., 2009; Lee et al., 2012; Liu et al., 2013) and porcine (Emmert et al., 2013b) myocardial infarction models resulted in greater heart function improvement compared with MSC suspensions. In an acute myocardial infarction rat model, Wang C.-C. et al. (2009) performed intramyocardial injection of $5.0 \times 10^{5} \mathrm{BM}$-derived MSC spheroids or MSC suspension and evaluated the echocardiography and catheterization measurements 4,8 , and 12 weeks post-operatively. The results showed superior heart function and stimulation of significant increase in vascular density for the MSC spheroid group (Wang C.-C. et al., 2009). In a delicate study, in vivo tracking of Dil-labeled UC-derived MSC spheroids showed that they can be differentiated into endothelial and cardiomyocyte cells at 4 weeks post-intramyocardial injection in a rat myocardial infarction model. At 7 weeks, the therapeutic efficacy of UCderived MSC spheroids is superior to MSC suspension in post-infarction left ventricular remodeling (Lee et al., 2012). Importantly, Liu et al. (2013) showed that adipose-derived MSC spheroids generated on chitosan membranes show a 20 -fold increase in cardiac marker gene expression (Gata4, Nkx2-5, Myh6, and Tnnt2) compared with MSC suspension cultures. In a similar approach, intramyocardial injection of $1 \times 10^{7}$ adipose-derived MSC spheroids in a rat myocardial infarction model showed better functional recovery compared with MSC suspensions after 12 weeks (Liu et al., 2013). Interestingly, a previous study indicated that intramyocardial injection of MSC spheroids consisting of adipose-derived MSC/human umbilical vein endothelial cells results in low arrhythmogenic potential but no further beneficial effects compared to the untreated group in a rat myocardial infarction model (Kolettis et al., 2018). In a larger animal model study, adipose-derived MSC were first labeled with micron-sized iron oxide particles, and then $2 \times 10^{7}$ MSC spheroids or MSC suspension were intra-myocardial injected in the porcineinfarcted myocardium. Moreover, the MSC spheroid engrafted successfully in $88.8 \%$ of animals keeping intact their micro architecture in vivo, whereas no arrhythmogenic, embolic, or neurological events occurred in the treated groups for up to 5 weeks follow-up (Emmert et al., 2013b). Therefore, preclinical studies established the feasibility, safety, and beneficial effects of intra-myocardial injected MSC spheroids in infarcted myocardium.

\section{NEOVASCULARIZATION AND ISCHEMIA}

In conjunction with the beneficial trophic effects of MSC spheroids toward infarcted myocardium, their applicability has been also investigated for neovascularization in vivo. In a mouse hind limb ischemia model, $1.0 \times 10^{7}$ cord-blood MSC 
spheroid intramuscular injection significantly increased the number of microvessels and $\alpha$ SMA-positive vessels, resulting in decreased fibrosis in the ischemic region, and attenuated limb loss and necrosis. In comparison, the MSC spheroid group showed a limb salvage rate of $75 \%$, whereas the MSC suspension group resulted in limb salvage rate of only $12.5 \%$ (Bhang et al., 2012). Additionally, Lee et al. (2016) showed that intramuscular injected adipose-derived MSC spheroids showed better proliferation than MSC suspension in the ischemic region, an effect that can be attributed to an increased expression of the proliferation marker PCNA. Therefore, MSC spheroids promote vascularization through secretion of angiogenic cytokines, preservation of ECM, and regulation of apoptotic signals.

\section{LIVER AND KIDNEY DISEASE}

The potential of MSC spheroids has been also investigated in liver regeneration and kidney injury models. For liver regeneration, two animal models have been tested for hepatectomy and CCl4-induced acute liver failure. In a pioneering study, Liu and Chang (2006) injected intraperitoneally $3 \times 10^{7} \mathrm{BM}$ MSC or hepatocytes in alginate-polylysine-alginate spheroids or suspension formats to treat $90 \%$ of hepatectomized rats. Up to day 14 , in the BM-MSC spheroid, hepatocyte spheroid, and hepatocyte suspension groups, most rats survived (83-100\%) and showed increased liver wet weight. Interestingly, these beneficial effects could be attributed to the increased expression in MSC spheroids of hepatocyte markers cytokeratin 8, cytokeratin 18, albumin, and $\alpha$-fetoprotein (Liu and Chang, 2006). In an improved approach, $3 \times 10^{7}$ BM-MSC spheroids or MSC suspension were intrasplenically injected to treat $90 \%$ of hepatectomized rats. On day 14, survival rate in MSC spheroid group was prolonged by almost $70 \%$ compared with the MSC suspension group via the secretion of hepatotrophic factors such as HGF and IL-6 into the liver. Of note, authors reported that implanted MSC may transdifferentiate into hepatocyte-like cells in vivo and therefore may render spleen as an ectopic functional liver support (Liu and Chang, 2009, 2012). This hypothesis has to be further investigated as MSC differentiation toward endodermal fate has not been widely established. In a CCl4-induced acute liver failure mouse model, $1 \times 10^{6}$ UC-MSC spheroids or MSC suspension were infused via the tail vein and, at day 2, resulted in liver injury attenuation. Specifically, MSC spheroids could promote IL-6 and IFN- $\gamma$ secretion but suppress TNF- $\alpha$ serum levels, and therefore significantly reduce tissue necrosis and increase liver regeneration ( $\mathrm{Li}$ et al., 2015). In a recent study, adipose-derived MSC spheroids have been used to treat an ischemia-reperfusion (I/R)-induced acute kidney injury rat model. Moreover, $2 \times 10^{6}$ MSC spheroids or MSC suspension were directly injected to the kidney cortex, and renal function was investigated for a 14-day follow-up. Results indicated that MSC spheroids are more beneficial to the kidney by reduction of tissue damage, increased vascularization, and amelioration of renal function compared with MSC suspensions. In detail, the MSC spheroid group showed increased levels of VEGF, HGF, and TSG-6 cytokines, and decreased levels of creatinine and blood urea nitrogen in the serum ( $\mathrm{Xu}$ et al., 2016). Therefore, in both liver and kidney injury animal models, MSC spheroid paracrine actions result in improved therapeutic effects characterized by reduced tissue necrosis, increased tissue regeneration, and improved organ function.

\section{FUTURE CLINICAL PERSPECTIVES}

To date, only a limited number of comparative preclinical studies have been performed between MSC spheroids and MSC suspension after 2D culture, whereas no clinical trials exist to evaluate the efficacy of MSC spheroids in clinical settings. As a result, there are no specific criteria to define when MSC spheroids would be preferable over MSC suspension to treat various clinical indications. However, it has become increasingly clear that current conventional and extensive 2D MSC culturing methods, similar to the ones used in public and commercial stem cell biobanks, even though they can ensure the generation of clinically relevant cell numbers for in vivo applications, cannot guarantee the preservation of MSC qualitative characteristics and their related high functionality. To circumvent these limitations, the incorporation of 3D MSC culturing approach into cell-based therapy would significantly impact the field, as more reproducible clinical outcomes may be achieved without requiring extensive ex vivo MSC manipulation and MSC stimulatory regimes (reviewed in Kouroupis et al., 2018). Specifically, current data indicate that MSC spheroid cultures with or without the usage of biomaterials not only preserve MSC phenotypic and molecular profiles but also significantly reinforce MSC functionality related to their immunomodulatory, anti-fibrotic, angiogenic, and trophic properties. In addition, as initial MSC homing and survival are crucial factors affecting the therapeutic outcome, 3D spheroid formation closely recapitulates the in vivo MSC niche, protect MSC viability, and works as a "vehicle" for their effective homing to the affected tissues upon implantation in vivo. On this basis, the adaptation of high-throughput regulatory-compliant and reproducible methods for MSC spheroid production would allow their use in clinical settings and contribute to an improved MSC-based product for safer and more effective therapeutic applications.

\section{AUTHOR CONTRIBUTIONS}

Both authors have made substantial contributions to the drafting of the article or revising it critically and to the final approval of the version to be submitted.

\section{ACKNOWLEDGMENTS}

We are in gratitude to the Soffer Family Foundation and the DRI Foundation for their generous funding support. 


\section{REFERENCES}

Agarwal, S. K., and Brenner, M. B. (2006). Role of adhesion molecules in synovial inflammation. Curr. Opin. Rheumatol. 18, 268-276. doi: 10.1097/01. bor.0000218948.42730.39

Aggarwal, S., and Pittenger, M. F. (2005). Human mesenchymal stem cells modulate allogeneic immune cell responses. Blood 105, 1815-1822.

Alimperti, S., and Andreadis, S. T. (2015). CDH2 and CDH11 act as regulators of stem cell fate decisions. Stem Cell Res. 14, 270-282. doi: 10.1016/j.scr.2015.02. 002

Alvarez-Pérez, J., Ballesteros, P., and Cerdán, S. (2005). Microscopic images of intraspheroidal $\mathrm{pH}$ by $1 \mathrm{H}$ magnetic resonance chemical shift imaging of $\mathrm{pH}$ sensitive indicators. Magma 18, 293-301. doi: 10.1007/s10334-005-0013-z

Amos, P. J., Kapur, S. K., Stapor, P. C., Shang, H., Bekiranov, S., Khurgel, M., et al. (2009). Human adipose-derived stromal cells accelerate diabetic wound healing: impact of cell formulation and delivery. Tissue Eng. Part A 16, 1595-1606. doi: 10.1089/ten.tea.2009.0616

Ayan, B., Heo, D. N., Zhang, Z., Dey, M., Povilianskas, A., Drapaca, C., et al. (2020). Aspiration-assisted bioprinting for precise positioning of biologics. Sci. Adv. 6:eaaw5111. doi: 10.1126/sciadv.aaw5111

Baraniak, P. R., Cooke, M. T., Saeed, R., Kinney, M. A., Fridley, K. M., and McDevitt, T. C. (2012). Stiffening of human mesenchymal stem cell spheroid microenvironments induced by incorporation of gelatin microparticles. J. Mech. Behav. Biomed. Mater. 11, 63-71. doi: 10.1016/j.jmbbm.2012.02.018

Bartosh, T. J., Ylöstalo, J. H., Bazhanov, N., Kuhlman, J., and Prockop, D. J. (2013). Dynamic compaction of human mesenchymal stem/precursor cells into spheres self-activates caspase-dependent IL1 signaling to enhance secretion of modulators of inflammation and immunity (PGE2, TSG6, and STC1). Stem Cells 31, 2443-2456. doi: 10.1002/stem.1499

Bartosh, T. J., Ylostalo, J. H., Mohammadipoor, A., Bazhanov, N., Coble, K., Claypool, K., et al. (2010). Aggregation of human mesenchymal stromal cells (MSCs) into 3D spheroids enhances their antiinflammatory properties. Proc. Natl. Acad. Sci. U.S.A. 107, 13724-13729. doi: 10.1073/pnas.1008117107

Bernardo, M. E., and Fibbe, W. E. (2013). Mesenchymal stromal cells: sensors and switchers of inflammation. Cell Stem Cell 13, 392-402. doi: 10.1016/j.stem.2013. 09.006

Bhang, S. H., Cho, S.-W., La, W.-G., Lee, T.-J., Yang, H. S., Sun, A.-Y., et al. (2011). Angiogenesis in ischemic tissue produced by spheroid grafting of human adipose-derived stromal cells. Biomaterials 32, 2734-2747. doi: 10.1016/ j.biomaterials.2010.12.035

Bhang, S. H., Lee, S., Shin, J.-Y., Lee, T.-J., and Kim, B.-S. (2012). Transplantation of cord blood mesenchymal stem cells as spheroids enhances vascularization. Tissue Eng. Part A 18, 2138-2147. doi: 10.1089/ten.tea.2011.0640

Blakely, A. M., Manning, K. L., Tripathi, A., and Morgan, J. R. (2015). Bio-pick, place, and perfuse: a new instrument for three-dimensional tissue engineering. Tissue Eng. Part C Methods 21, 737-746. doi: 10.1089/ten.TEC.2014.0439

Bulanova, E. A., Koudan, E. V., Degosserie, J., Heymans, C., Pereira, F. D., Parfenov, V. A., et al. (2017). Bioprinting of a functional vascularized mouse thyroid gland construct. Biofabrication 9:034105. doi: 10.1088/1758-5090/aa7fdd

Caplan, A. I. (2017). mesenchymal stem cells: time to change the name! Stem Cells Transl. Med. 6, 1445-1451. doi: 10.1002/sctm.17-0051

Caplan, A. I., and Correa, D. (2011). The MSC: an injury drugstore. Cell Stem Cell 9, 11-15.

Cechin, S., Alvarez-Cubela, S., Giraldo, J. A., Molano, R. D., Villate, S., Ricordi, C., et al. (2014). Influence of in vitro and in vivo oxygen modulation on $\beta$ cell differentiation from human embryonic stem cells. Stem Cells Transl. Med. 3, 277-289. doi: 10.5966/sctm.2013-0160

Centeno, C. J., Busse, D., Kisiday, J., Keohan, C., Freeman, M., and Karli, D. (2008). Regeneration of meniscus cartilage in a knee treated with percutaneously implanted autologous mesenchymal stem cells. Med. Hypotheses 71, 900-908. doi: 10.1016/j.mehy.2008.06.042

Chang, S. K., Noss, E. H., Chen, M., Gu, Z., Townsend, K., Grenha, R., et al. (2011). Cadherin-11 regulates fibroblast inflammation. Proc. Natl.Acad. Sci. U.S.A. 108:8402. doi: 10.1073/pnas.1019437108

Colle, J., Blondeel, P., Bruyne, A., Bochar, S., Tytgat, L., Vercruysse, C., et al. (2020). Bioprinting predifferentiated adipose-derived mesenchymal stem cell spheroids with methacrylated gelatin ink for adipose tissue engineering. J. Mater. Sci. 31:36. doi: 10.1007/s10856-020-06374-w
Cui, X., Hartanto, Y., and Zhang, H. (2017). Advances in multicellular spheroids formation. J. R. Soc. Interface 14:20160877. doi: 10.1098/rsif.2016.0877

Curcio, E., Salerno, S., Barbieri, G., De Bartolo, L., Drioli, E., and Bader, A. (2007). Mass transfer and metabolic reactions in hepatocyte spheroids cultured in rotating wall gas-permeable membrane system. Biomaterials 28, 5487-5497. doi: 10.1016/j.biomaterials.2007.08.033

da Silva Meirelles, L., Fontes, A. M., Covas, D. T., and Caplan, A. I. (2009). Mechanisms involved in the therapeutic properties of mesenchymal stem cells. Cytokine Growth Factor Rev. 20, 419-427. doi: 10.1016/j.cytogfr.2009.10.002

De Bari, C., Dell'Accio, F., Tylzanowski, P., and Luyten, F. P. (2001). Multipotent mesenchymal stem cells from adult human synovial membrane. Arthritis Rheumatism 44, 1928-1942.

de Bournonville, S., Lambrechts, T., Vanhulst, J., Luyten, F. P., Papantoniou, I., and Geris, L. (2019). Towards self-regulated bioprocessing: a compact benchtop bioreactor system for monitored and controlled 3D cell and tissue culture. Biotechnol. J. 14:1800545. doi: 10.1002/biot.201800545

De Moor, L., Fernandez, S., Vercruysse, C., Tytgat, L., Asadian, M., De Geyter, N., et al. (2020). Hybrid bioprinting of chondrogenically induced human mesenchymal stem cell spheroids. Front. Bioeng. Biotechnol. 8:484. doi: 10.3389/ fbioe.2020.00484

Deynoux, M., Sunter, N., Ducrocq, E., Dakik, H., Guibon, R., Burlaud-Gaillard, J., et al. (2020). A comparative study of the capacity of mesenchymal stromal cell lines to form spheroids. PLoS One 15:e0225485. doi: 10.1371/journal.pone. 0225485

Doucet, C., Ernou, I., Zhang, Y., Llense, J.-R., Begot, L., Holy, X., et al. (2005). Platelet lysates promote mesenchymal stem cell expansion: a safety substitute for animal serum in cell-based therapy applications. J. Cell. Physiol. 205, 228-236. doi: 10.1002/jcp.20391

Dragoo, J. L., Samimi, B., Zhu, M.-L., Hame, S. L., Thomas, B. L., Lieberman, J. R., et al. (2003). Tissue-engineered cartilage and bone using stem cells from human infrapatellar fat pads. J. Bone Joint Surg. Br. 85, 740-747.

Emmert, M. Y., Wolint, P., Wickboldt, N., Gemayel, G., Weber, B., Brokopp, C. E., et al. (2013a). Human stem cell-based three-dimensional microtissues for advanced cardiac cell therapies. Biomaterials 34, 6339-6354. doi: 10.1016/ j.biomaterials.2013.04.034

Emmert, M. Y., Wolint, P., Winklhofer, S., Stolzmann, P., Cesarovic, N., Fleischmann, T., et al. (2013b). Transcatheter based electromechanical mapping guided intramyocardial transplantation and in vivo tracking of human stem cell based three dimensional microtissues in the porcine heart. Biomaterials 34, 2428-2441. doi: 10.1016/j.biomaterials.2012.12.021

Foty, R. A., and Steinberg, M. S. (2005). The differential adhesion hypothesis: a direct evaluation. Dev. Biol. 278, 255-263. doi: 10.1016/j.ydbio.2004.11.012

Foty, R. (2011). A simple hanging drop cell culture protocol for generation of 3D spheroids. J. Vis. Exp. 51, 2720. doi: 10.3791/2720

Fraker, C. A., Alvarez, S., Papadopoulos, P., Giraldo, J., Gu, W., Ricordi, C., et al. (2007). Enhanced oxygenation promotes beta-cell differentiation in vitro. Stem Cells 25, 3155-3164. doi: 10.1634/stemcells.2007-0445

Fraker, C. A., Cechin, S., Álvarez-Cubela, S., Echeverri, F., Bernal, A., Poo, R., et al. (2013). A physiological pattern of oxygenation using perfluorocarbonbased culture devices maximizes pancreatic islet viability and enhances $\beta$ Cell function. Cell Transplantation 22, 1723-1733. doi: 10.3727/096368912X65 7873

Friedenstein, A. J., Chailakhyan, R. K., Latsinik, N. V., Panasyuk, A. F., and Keiliss-Borok, I. V. (1974). Stromal cells responsible for transferring the microenvironment of the hemopoietic tissues. Cloning in vitro and retransplantation in vivo. Transplantation 17, 331-340.

Frith, J. E., Thomson, B., and Genever, P. G. (2010). Dynamic three-dimensional culture methods enhance mesenchymal stem cell properties and increase therapeutic potential. Tissue Eng. Part C Methods 16, 735-749. doi: 10.1089/ ten.TEC.2009.0432

Guilak, F., Estes, B. T., Diekman, B. O., Moutos, F. T., and Gimble, J. M. (2010). 2010 nicolas andry award: multipotent adult stem cells from adipose tissue for musculoskeletal tissue engineering. Clin. Orthopaedics Relat. Res. 468, 2530-2540. doi: 10.1007/s11999-010-1410-9

Gutzweiler, L., Kartmann, S., Troendle, K., Benning, L., Finkenzeller, G., Zengerle, R., et al. (2017). Large scale production and controlled deposition of single HUVEC spheroids for bioprinting applications. Biofabrication 9:025027. doi: $10.1088 / 1758-5090 /$ aa 7218 
Hass, R., Kasper, C., Böhm, S., and Jacobs, R. (2011). Different populations and sources of human mesenchymal stem cells (MSC): a comparison of adult and neonatal tissue-derived MSC. Cell Commun. Signal. 9:12. doi: 10.1186/1478811X-9- 12

Hsu, S.-H., and Hsieh, P.-S. (2015). Self-assembled adult adipose-derived stem cell spheroids combined with biomaterials promote wound healing in a rat skin repair model. Wound Repair Regen. 23, 57-64. doi: 10.1111/wrr.12239

Hsu, S. H., and Huang, G. S. (2013). Substrate-dependent Wnt signaling in MSC differentiation within biomaterial-derived 3D spheroids. Biomaterials 34, 4725-4738. doi: 10.1016/j.biomaterials.2013.03.031

Huang, G. S., Dai, L. G., Yen, B. L., and Hsu, S. H. (2011). Spheroid formation of mesenchymal stem cells on chitosan and chitosan-hyaluronan membranes. Biomaterials 32, 6929-6945. doi: 10.1016/j.biomaterials.2011.05.092

Ip, B. C., Cui, F., Tripathi, A., and Morgan, J. R. (2016). The bio-gripper: a fluid-driven micro-manipulator of living tissue constructs for additive bio-manufacturing. Biofabrication 8:025015. doi: 10.1088/1758-5090/8/2/02 5015

Jakab, K., Norotte, C., Damon, B., Marga, F., Neagu, A., Besch-Williford, C. L., et al. (2008). Tissue engineering by self-assembly of cells printed into topologically defined structures. Tissue Eng. Part A 14, 413-421. doi: 10.1089/tea.2007.0173

Jones, E., Crawford, A., English, A., Henshaw, K., Mundy, J., Corscadden, D., et al. (2008). Synovial fluid mesenchymal stem cells in health and early osteoarthritis: detection and functional evaluation at the single-cell level. Arthritis Rheumatism 58, 1731-1740.

Jung, S., Sen, A., Rosenberg, L., and Behie, L. A. (2010). Identification of growth and attachment factors for the serum-free isolation and expansion of human mesenchymal stromal cells. Cytotherapy 12, 637-657.

Karnieli, O., Friedner, O. M., Allickson, J. G., Zhang, N., Jung, S., Fiorentini, D., et al. (2017). A consensus introduction to serum replacements and serum-free media for cellular therapies. Cytotherapy 19, 155-169. doi: 10.1016/j.jcyt.2016. 11.011

Koç, O. N., Day, J., Nieder, M., Gerson, S. L., Lazarus, H. M., and Krivit, W. (2002). Allogeneic mesenchymal stem cell infusion for treatment of metachromatic leukodystrophy (MLD) and Hurler syndrome (MPS-IH). Bone Marrow Transplant 30, 215-222. doi: 10.1038/sj.bmt.1703650

Kolettis, T. M., Bagli, E., Barka, E., Kouroupis, D., Kontonika, M., Vilaeti, A. D., et al. (2018). Medium-term electrophysiologic effects of a cellularized scaffold implanted in rats after myocardial infarction. Cureus 10:e2959. doi: 10.7759/ cureus.2959

Kouroupis, D., Bowles, A. C., Best, T. M., Kaplan, L. D., and Correa, D. (2020a). CD10/Neprilysin enrichment in infrapatellar fat pad-derived mesenchymal stem cells under regulatory-compliant conditions: implications for efficient synovitis and fat pad fibrosis reversal. Am. J. Sports Med. 48, 2013-2027. doi: $10.1177 / 0363546520917699$

Kouroupis, D., Bowles, A. C., Greif, D. N., Leñero, C., Best, T. M., Kaplan, L. D., et al. (2020b). Regulatory-compliant conditions during cell product manufacturing enhance in vitro immunomodulatory properties of infrapatellar fat pad-derived mesenchymal stem/stromal cells. Cytotherapy 22, 677-689. doi: 10.1016/j.jcyt.2020.06.007

Kouroupis, D., Sanjurjo-Rodriguez, C., Jones, E., and Correa, D. (2018). Mesenchymal stem cell functionalization for enhanced therapeutic applications. Tissue Eng. Part B 25, 55-77. doi: 10.1089/ten.teb.2018.0118

Kouroupis, D., Wang, X. N., El-Sherbiny, Y., McGonagle, D., and Jones, E. (2017). "The safety of non-expanded multipotential stromal cell therapies," in Safety, Ethics and Regulations, eds P. V. Pham and A. Rosemann (Cham: Springer International Publishing), 91-118.

Kouroupis, D., Willman, M. A., Best, T. M., Kaplan, L. D., and Correa, D. (2021). Infrapatellar fat pad-derived mesenchymal stem cell-based spheroids enhance their therapeutic efficacy to reverse synovitis and fat pad fibrosis. Stem Cell Res. Ther. 12:44. doi: 10.1186/s13287-020-02107-6

Krampera, M., Galipeau, J., Shi, Y., Tarte, K., and Sensebe, L. (2013). Immunological characterization of multipotent mesenchymal stromal cellsThe international society for cellular therapy (ISCT) working proposal. Cytotherapy 15, 1054-1061. doi: 10.1016/j.jcyt.2013.02.010

Le Blanc, K., Frassoni, F., Ball, L., Locatelli, F., Roelofs, H., Lewis, I., et al. (2008). Mesenchymal stem cells for treatment of steroid-resistant, severe, acute graft-versus-host disease: a phase II study. Lancet 371, 1579-1586.
Lee, E. J., Park, S. J., Kang, S. K., Kim, G.-H., Kang, H.-J., Lee, S.-W., et al. (2012). Spherical bullet formation via e-cadherin promotes therapeutic potency of mesenchymal stem cells derived from human umbilical cord blood for myocardial infarction. Mol. Ther. 20, 1424-1433. doi: 10.1038/mt.2012.58

Lee, J. H., Han, Y.-S., and Lee, S. H. (2016). Long-duration threedimensional spheroid culture promotes angiogenic activities of adipose-derived mesenchymal stem cells. Biomol. Ther. 24, 260-267. doi: 10.4062/biomolther.2015.146

Lewis, E. E. L., Wheadon, H., Lewis, N., Yang, J., Mullin, M., Hursthouse, A., et al. (2016). A quiescent, regeneration-responsive tissue engineered mesenchymal stem cell bone marrow niche model via magnetic levitation. ACS Nano 10, 8346-8354. doi: 10.1021/acsnano.6b02841

Li, Y., Guo, G., Li, L., Chen, F., Bao, J., Shi, Y.-J., et al. (2015). Three-dimensional spheroid culture of human umbilical cord mesenchymal stem cells promotes cell yield and stemness maintenance. Cell Tissue Res. 360, 297-307. doi: 10.1007/ s00441-014-2055-X

Liu, B.-H., Yeh, H.-Y., Lin, Y.-C., Wang, M.-H., Chen, D. C., Lee, B.-H., et al. (2013). Spheroid formation and enhanced cardiomyogenic potential of adiposederived stem cells grown on chitosan. BioResearch Open Access 2, 28-39. doi: 10.1089/biores.2012.0285

Liu, Z. C., and Chang, T. M. S. (2006). Transdifferentiation of bioencapsulated bone marrow cells into hepatocyte-like cells in the $90 \%$ hepatectomized rat model. Liver Transplantation 12, 566-572. doi: 10.1002/lt.20635

Liu, Z. C., and Chang, T. M. S. (2009). Preliminary study on intrasplenic implantation of artificial cell bioencapsulated stem cells to increase the survival of 90\% hepatectomized rats. Artif. Cells Blood Subst. Biotechnol. 37, 53-55. doi: 10.1080/10731190802663975

Liu, Z. C., and Chang, T. M. S. (2012). Intrasplenic transplantation of bioencapsulated mesenchymal stem cells improves the recovery rates of $90 \%$ partial hepatectomized rats. Stem Cells Int. 2012:697094. doi: 10.1155/2012/ 697094

Lou, Y., Guo, D., Zhang, H., and Song, L. (2016). Effectiveness of mesenchymal stems cells cultured by hanging drop vs. conventional culturing on the repair of hypoxic-ischemic-damaged mouse brains, measured by stemness gene expression. Open Life Sci. 11, 519-523.

Ma, D., Zhong, C., Yao, H., Liu, Y., Chen, F., Li, J., et al. (2011). Engineering injectable bone using bone marrow stromal cell aggregates. Stem Cells Dev. 20, 989-999. doi: 10.1089/scd.2010.0348

Mackay, A. M., Beck, S. C., Murphy, J. M., Barry, F. P., Chichester, C. O., and Pittenger, M. F. (1998). Chondrogenic differentiation of cultured human mesenchymal stem cells from marrow. Tissue Eng. 4, 415-428. doi: 10.1089/ten. 1998.4.415

Markou, M., Kouroupis, D., Badounas, F., Katsouras, A., Kyrkou, A., Fotsis, T., et al. (2020). Tissue engineering using vascular organoids from human pluripotent stem cell derived mural cell phenotypes. Front. Bioeng. Biotechnol. 8:278. doi: 10.3389/fbioe.2020.00278

Mekhileri, N. V., Lim, K. S., Brown, G. C. J., Mutreja, I., Schon, B. S., Hooper, G. J., et al. (2018). Automated 3D bioassembly of micro-tissues for biofabrication of hybrid tissue engineered constructs. Biofabrication 10:024103. doi: 10.1088/ 1758-5090/aa9ef1

Menard, C., Pacelli, L., Bassi, G., Dulong, J., Bifari, F., Bezier, I., et al. (2013). Clinical-grade mesenchymal stromal cells produced under various good manufacturing practice processes differ in their immunomodulatory properties: standardization of immune quality controls. Stem Cells Dev. 22, 1789-1801. doi: $10.1089 /$ scd.2012.0594

Mendicino, M., Bailey, Alexander, M., Wonnacott, K., Puri, R. K., Bauer, et al. (2014). MSC-based product characterization for clinical trials: an FDA perspective. Cell Stem Cell 14, 141-145. doi: 10.1016/j.stem.2014.01.013

Meng, R., Xu, H.-Y., Di, S.-M., Shi, D.-Y., Qian, A.-R., Wang, J.-F., et al. (2011). Human mesenchymal stem cells are sensitive to abnormal gravity and exhibit classic apoptotic features. Acta Bioch. Biophys. Sin. 43, 133-142. doi: 10.1093/ abbs/gmq121

Messina, A., Morelli, S., Forgacs, G., Barbieri, G., Drioli, E., and De Bartolo, L. (2017). Self-assembly of tissue spheroids on polymeric membranes. J. Tissue Eng. Regen. Med. 11, 2090-2103. doi: 10.1002/term.2105

Meyers, V. E., Zayzafoon, M., Douglas, J. T., and McDonald, J. M. (2005). RhoA and cytoskeletal disruption mediate reduced osteoblastogenesis and enhanced 
adipogenesis of human mesenchymal stem cells in modeled microgravity. J. Bone Min. Res. 20, 1858-1866. doi: 10.1359/JBMR.050611

Meyers, V. E., Zayzafoon, M., Gonda, S. R., Gathings, W. E., and McDonald, J. M. (2004). Modeled microgravity disrupts collagen I/integrin signaling during osteoblastic differentiation of human mesenchymal stem cells. J. Cell. Biochem. 93, 697-707. doi: 10.1002/jcb.20229

Mironov, V., Visconti, R. P., Kasyanov, V., Forgacs, G., Drake, C. J., and Markwald, R. R. (2009). Organ printing: tissue spheroids as building blocks. Biomaterials 30, 2164-2174. doi: 10.1016/j.biomaterials.2008.12.084

Moldovan, N. I., Hibino, N., and Nakayama, K. (2017). Principles of the kenzan method for robotic cell spheroid-based three-dimensional bioprinting. Tissue Eng. Part B Rev. 23, 237-244. doi: 10.1089/ten.TEB.2016.0322

Moretti, P., Hatlapatka, T., Marten, D., Lavrentieva, A., Majore, I., Hass, R., et al. (2010). Mesenchymal stromal cells derived from human umbilical cord tissues: primitive cells with potential for clinical and tissue engineering applications. Adv. Biochem. Eng. Biotechnol. 123, 29-54. doi: 10.1007/10_20 09_15

Mueller-Klieser, W. (1984). Method for the determination of oxygen consumption rates and diffusion coefficients in multicellular spheroids. Biophys. J. 46, 343348. doi: 10.1016/s0006-3495(84)84030-8

Murphy, K. C., Fang, S. Y., and Leach, J. K. (2014). Human mesenchymal stem cell spheroids in fibrin hydrogels exhibit improved cell survival and potential for bone healing. Cell Tissue Res. 357, 91-99. doi: 10.1007/s00441-014$1830-\mathrm{z}$

Murphy, K. C., Hung, B. P., Browne-Bourne, S., Zhou, D., Yeung, J., Genetos, D. C., et al. (2017). Measurement of oxygen tension within mesenchymal stem cell spheroids. J. R. Soc. Interface 14:20160851. doi: 10.1098/rsif.2016.0851

Nikolova, M. P., and Chavali, M. S. (2019). Recent advances in biomaterials for 3D scaffolds: a review. Bioact. Mater. 4, 271-292. doi: 10.1016/j.bioactmat.2019.10. 005

Petrenko, Y., Syková, E., and Kubinová, Š (2017). The therapeutic potential of three-dimensional multipotent mesenchymal stromal cell spheroids. Stem Cell Res. Ther. 8:94. doi: 10.1186/s13287-017-0558-6

Pittenger, M. F., Discher, D. E., Péault, B. M., Phinney, D. G., Hare, J. M., and Caplan, A. I. (2019). Mesenchymal stem cell perspective: cell biology to clinical progress. npj Regen. Med. 4:22. doi: 10.1038/s41536-019-0083-6

Potapova, I. A., Brink, P. R., Cohen, I. S., and Doronin, S. V. (2008). Culturing of human mesenchymal stem cells as three-dimensional aggregates induces functional expression of CXCR4 that regulates adhesion to endothelial cells. J. Biol. Chem. 283, 13100-13107. doi: 10.1074/jbc.M80018 4200

Potapova, I. A., Gaudette, G. R., Brink, P. R., Robinson, R. B., Rosen, M. R., Cohen, I. S., et al. (2007). Mesenchymal stem cells support migration, extracellular matrix invasion, proliferation, and survival of endothelial cells in vitro. Stem Cells 25, 1761-1768.

Redondo-Castro, E., Cunningham, C. J., Miller, J., Brown, H., Allan, S. M., and Pinteaux, E. (2018a). Changes in the secretome of tri-dimensional spheroidcultured human mesenchymal stem cells in vitro by interleukin-1 priming. Stem Cell Res. Ther. 9:11. doi: 10.1186/s13287-017-0753-5

Redondo-Castro, E., Cunningham, C. J., Miller, J., Cain, S. A., Allan, S. M., and Pinteaux, E. (2018b). Generation of Human mesenchymal stem cell 3D spheroids using low-binding plates. Bio Protoc. 8:e2968. doi: 10.21769/ BioProtoc. 2968

Ringden, O., Uzunel, M., Rasmusson, I., Remberger, M., Sundberg, B., Lonnies, H., et al. (2006). Mesenchymal stem cells for treatment of therapy-resistant graft-versus-host disease. Transplantation 81, 1390-1397.

Sart, S., Tsai, A. C., Li, Y., and Ma, T. (2014). Three-dimensional aggregates of mesenchymal stem cells: cellular mechanisms, biological properties, and applications. Tissue Eng. Part B Rev. 20, 365-380. doi: 10.1089/ten.TEB.2013. 0537

Seda Tiğlı, R., Karakeçili, A., and Gümüșderelioğlu, M. (2007). In vitro characterization of chitosan scaffolds: influence of composition and deacetylation degree. J. Mater. Sci.18, 1665-1674. doi: 10.1007/s10856007-3066-x

Sheyn, D., Pelled, G., Netanely, D., Domany, E., and Gazit, D. (2010). The effect of simulated microgravity on human mesenchymal stem cells cultured in an osteogenic differentiation system: a bioinformatics study. Tissue Eng Part A 16, 3403-3412. doi: 10.1089/ten.tea.2009.0834
Singer, N. G., and Caplan, A. I. (2011). Mesenchymal stem cells: mechanisms of inflammation. Annu. Rev. Pathol. 6, 457-478. doi: 10.1146/annurev-pathol011110-130230

Sorrell, J. M., Baber, M. A., and Caplan, A. I. (2009). Influence of adult mesenchymal stem cells on in vitro vascular formation. Tissue Eng Part A 15, 1751-1761. doi: 10.1089/ten.tea.2008.0254

Suenaga, H., Furukawa, K. S., Suzuki, Y., Takato, T., and Ushida, T. (2015). Bone regeneration in calvarial defects in a rat model by implantation of human bone marrow-derived mesenchymal stromal cell spheroids. J. Mater. Sci. Mater. Med. 26:254. doi: 10.1007/s10856-015-5591-3

Suzuki, S., Muneta, T., Tsuji, K., Ichinose, S., Makino, H., Umezawa, A., et al. (2012). Properties and usefulness of aggregates of synovial mesenchymal stem cells as a source for cartilage regeneration. Arthritis Res. Ther. 14:R136.

Theisen, C. S., Wahl, J. K. III, Johnson, K. R., and Wheelock, M. J. (2007). NHERF links the N-cadherin/catenin complex to the platelet-derived growth factor receptor to modulate the actin cytoskeleton and regulate cell motility. Mol. Biol. Cell 18, 1220-1232. doi: 10.1091/mbc.e06-10-0960

Toma, C., Pittenger Mark, F., Cahill Kevin, S., Byrne Barry, J., and Kessler Paul, D. (2002). Human mesenchymal stem cells differentiate to a cardiomyocyte phenotype in the adult murine heart. Circulation 105, 93-98. doi: 10.1161/ hc0102.101442

Toma, C., Wagner, W. R., Bowry, S., Schwartz, A., and Villanueva, F. (2009). Fate of culture-expanded mesenchymal stem cells in the microvasculature: in vivo observations of cell kinetics. Circ. Res. 104, 398-402. doi: 10.1161/ CIRCRESAHA.108.187724

Uccelli, A., and Rosbo, N. K. (2015). The immunomodulatory function of mesenchymal stem cells: mode of action and pathways. Ann. N. Y. Acad. Sci. 1351, 114-126. doi: 10.1111/nyas. 12815

Vorwald, C. E., Ho, S. S., Whitehead, J., and Leach, J. K. (2018). High-throughput formation of mesenchymal stem cell spheroids and entrapment in alginate hydrogels. Methods Mol. Biol. 1758, 139-149. doi: 10.1007/978-1-4939-77413_11

Wang, C.-C., Chen, C.-H., Hwang, S.-M., Lin, W.-W., Huang, C.-H., Lee, W.-Y., et al. (2009). Spherically symmetric mesenchymal stromal cell bodies inherent with endogenous extracellular matrices for cellular cardiomyoplasty. Stem Cells 27, 724-732. doi: 10.1634/stemcells.2008-0944

Wang, W., Itaka, K., Ohba, S., Nishiyama, N., Chung, U.-I., Yamasaki, Y., et al. (2009). 3D spheroid culture system on micropatterned substrates for improved differentiation efficiency of multipotent mesenchymal stem cells. Biomaterials 30, 2705-2715. doi: 10.1016/j.biomaterials.2009.01.030

Waterman, R. S., Tomchuck, S. L., Henkle, S. L., and Betancourt, A. M. (2010). A new mesenchymal stem cell (MSC) paradigm: polarization into a proinflammatory MSC1 or an immunosuppressive MSC2 phenotype. PLoS One 5:e10088. doi: 10.1371/journal.pone.0010088

Weiss, M., and Troyer, D. (2006). Stem cells in the umbilical cord. Stem Cell Rev. Rep. 2, 155-162.

Xu, L., Meng, F., Ni, M., Lee, Y., and Li, G. (2013). N-cadherin regulates osteogenesis and migration of bone marrow-derived mesenchymal stem cells. Mol. Biol. Rep. 40, 2533-2539. doi: 10.1007/s11033-0122334-0

Xu, Y., Shi, T., Xu, A., and Zhang, L. (2016). 3D spheroid culture enhances survival and therapeutic capacities of MSCs injected into ischemic kidney. J. Cell. Mol.Med. 20, 1203-1213. doi: $10.1111 / \mathrm{jcmm} .12651$

Yanagihara, K., Uchida, S., Ohba, S., Kataoka, K., and Itaka, K. (2018). Treatment of bone defects by transplantation of genetically modified mesenchymal stem cell spheroids. Mol. Ther. Methods Clin. Dev. 9, 358-366. doi: 10.1016/j.omtm. 2018.04.006

Yang, C. M., Huang, Y. J., and Hsu, S. H. (2015). Enhanced autophagy of adiposederived stem cells grown on chitosan substrates. Biores. Open Access 4, 89-96. doi: 10.1089/biores.2014.0032

Yeh, H.-Y., Liu, B.-H., and Hsu, S.-H. (2012). The calcium-dependent regulation of spheroid formation and cardiomyogenic differentiation for MSCs on chitosan membranes. Biomaterials 33, 8943-8954. doi: 10.1016/j.biomaterials.2012. 08.069

Yeh, H.-Y., Liu, B.-H., Sieber, M., and Hsu, S.-H. (2014). Substrate-dependent gene regulation of self-assembled human MSC spheroids on chitosan membranes. BMC Genomics 15:10. doi: 10.1186/1471-2164-15-10 
Ylöstalo, J. H., Bartosh, T. J., Coble, K., and Prockop, D. J. (2012). Human mesenchymal stem/stromal cells (hMSCs) cultured as spheroids are selfactivated to produce prostaglandin E2 (PGE2) that directs stimulated macrophages into an anti-inflammatory phenotype. Stem Cells 30, 2283-2296. doi: $10.1002 /$ stem. 1191

Ylostalo, J. H., Bartosh, T. J., Tiblow, A., and Prockop, D. J. (2014). Unique characteristics of human mesenchymal stromal/progenitor cells pre-activated in 3-dimensional cultures under different conditions. Cytotherapy 16, 1486-1500. doi: 10.1016/j.jcyt.2014.07.010

Ylostalo, J. H., Bazhanov, N., Mohammadipoor, A., and Bartosh, T. J. (2017). Production and administration of therapeutic mesenchymal stem/stromal cell (MSC) spheroids primed in 3-D cultures under xeno-free conditions. J. Vis. Exp. 121, 55126. doi: 10.3791/55126

Yu, B., Yu, D., Cao, L., Zhao, X., Long, T., Liu, G., et al. (2011). Simulated microgravity using a rotary cell culture system promotes chondrogenesis of human adipose-derived mesenchymal stem cells via the p38 MAPK pathway. Biochem. Biophys. Res. Commun. 414, 412-418. doi: 10.1016/j.bbrc.2011. 09.103

Zhang, K., Yan, S., Li, G., Cui, L., and Yin, J. (2015). In-situ birth of MSCs multicellular spheroids in poly(l-glutamic acid)/chitosan scaffold for hyalinelike cartilage regeneration. Biomaterials 71, 24-34. doi: 10.1016/j.biomaterials. 2015.08.037
Zhang, Q., Nguyen, A. L., Shi, S., Hill, C., Wilder-Smith, P., Krasieva, T. B., et al. (2012). Three-dimensional spheroid culture of human gingivaderived mesenchymal stem cells enhances mitigation of chemotherapyinduced oral mucositis. Stem Cells Dev. 21, 937-947. doi: 10.1089/scd.2011. 0252

Zimmermann, J. A., and McDevitt, T. C. (2014). Pre-conditioning mesenchymal stromal cell spheroids for immunomodulatory paracrine factor secretion. Cytotherapy 16, 331-345. doi: 10.1016/j.jcyt.2013.09.004

Zuk, P. A., Zhu, M., Ashjian, P., De Ugarte, D. A., Huang, J. I., Mizuno, H., et al. (2002). Human adipose tissue is a source of multipotent stem cells. Mol. Biol. Cell 13, 4279-4295.

Conflict of Interest: The authors declare that the research was conducted in the absence of any commercial or financial relationships that could be construed as a potential conflict of interest.

Copyright (c) 2021 Kouroupis and Correa. This is an open-access article distributed under the terms of the Creative Commons Attribution License (CC BY). The use, distribution or reproduction in other forums is permitted, provided the original author(s) and the copyright owner(s) are credited and that the original publication in this journal is cited, in accordance with accepted academic practice. No use, distribution or reproduction is permitted which does not comply with these terms. 